\title{
The effect of football boot upper padding on dribbling and passing performance using a test-retest validated protocol
}

\author{
Katrine Okholm Kryger ${ }^{1,2,3} \cdot$ Séan Mitchell ${ }^{1,2} \cdot$ Diwei Zhou $^{4} \cdot$ Steph Forrester ${ }^{1,2}$
}

Published online: 31 August 2018

(c) The Author(s) 2018

\begin{abstract}
Touch/control football boots are reportedly designed for optimal passing and dribbling. Little research exists on the effect of boot design on touch/control performance and no validated protocol has been developed for assessing passing and dribbling from an equipment focus. This study aimed to assess the effect of upper padding on dribbling and passing performance using a test-retest reliable test setup. Eight university players performed a protocol of dribbling, short and long passing in football boots with 0 and $6 \mathrm{~mm}$ of upper padding (Poron foam). The protocol was completed twice; the 0 -mm padding results were used for test-retest validation, while the $0-\mathrm{mm}$ versus $6-\mathrm{mm}$ padding results were used to investigate the effect of padding. Dribbling performance was assessed though completion time, number of touches applied and lateral deviation from cones and passing performance through ball velocity and offset from target. The protocol demonstrated good test-retest reliability and indicated no significant differences in any of the 12 performance variables between the 0 - and 6-mm padded boots. These findings suggest an element of design freedom in the use of padding within football boot uppers without affecting dribbling or passing performance.
\end{abstract}

Keywords Soccer $\cdot$ Footwear $\cdot$ Shoe $\cdot$ Precision $\cdot$ Accuracy $\cdot$ Reliability

\section{Introduction}

Sporting goods companies need to frequently introduce technological innovations to distinguish themselves in an increasingly competitive and dynamic market [1]. It is

Katrine Okholm Kryger

K.Kryger@qmul.ac.uk

Séan Mitchell

S.R.Mitchell@lboro.ac.uk

Diwei Zhou

D.Zhou2@lboro.ac.uk

Steph Forrester

S.Forrester@lboro.ac.uk

1 School of Mechanical, Electrical and Manufacturing Engineering, Loughborough University, Loughborough LE11 3TU, UK

2 Sports Technology Institute, Loughborough University, 1 Oakwood Dr, Loughborough LE11 3QF, UK

3 Sport and Exercise Medicine, Queen Mary University of London, London E1 4NS, UK

4 Department of Mathematical Sciences, Loughborough University, Epinal Way, Loughborough LE11 3TU, UK currently common practice to market football boots with an emphasis on enhancing a single key performance characteristic (e.g. running speed, touch/control or kicking power). Despite the fundamental importance of football boot design when delivering advertised performance benefits, little research has been published on how specific design parameters impact performance.

Boots with enhanced touch/control are reportedly designed for optimal passing and dribbling (e.g. PUMA EvoTouch, Nike Magista and Adidas Ace). These skills are acknowledged as an important aspect of the modern game with analysis of FA Premier League matches highlighting dribbling and short passes as the most frequently performed ball handling skills during match play [2]. The design of these football boots, however, follows no obvious visible trends. The PUMA EvoTouch Pro is designed with "Ultrathin K-Touch leather upper" using kangaroo leather [3]. The Nike Magista models have a thicker, stiffer textured upper with added localised padded pressure points and All Conditions Control technology [4]. The Adidas Ace 17+ is designed with a thinner, smoother, laceless sock forefoot, coated with a thin layer of raised NON STOP GRIP dots [5]. Design technologies claimed to improve touch/control are 
therefore wide ranging. All designs, however, focus on the football boot upper but companies apply different materials and padding choices. This may indicate that manufacturers do not have a clear vision of what an optimal design is for passing and dribbling.

Similarly, little research has assessed the effect of boot design on passing or dribbling performance. One study assessed passing and dribbling performance across four footwear conditions which included two football boots, an indoor court shoe and barefoot [6]. However, the two football boots varied in many design features making it difficult to assess the effect of any single feature. Furthermore, assessment relied on visual counting of ball touches during dribbling as well as visual detection of the ball landing position during the aerial passing drill which may give less reliable data than more objective measurement tools such as video digitisation.

Although, several methodologies have been developed and validated for passing and dribbling performance assessment in football, these have mainly been conceived to assess the effect of player level or nutritional interventions rather than boot performance [7-9]. Decision-making is a key focus when assessing human performance but should be minimised when assessing the effect of equipment, including boot design. Whilst dribbling and passing performance are multifactorial, past literature has tended to focus on a single measure of performance, e.g. time for dribbling or accuracy for passing [9]. Additionally, passing length varies from 2.5 to $36 \mathrm{~m}$ in the literature with no rationale provided for the chosen length. Therefore, an attempt to define an appropriate protocol for the assessment of dribbling and passing performance, applicable to the investigation of football boot design, is needed.

This study aimed to assess the effect of football boot upper padding on dribbling and passing performance through a multifactorial and controlled approach using a novel test protocol. The setup was structured to be easy to apply and demand no more than two researchers to run yet be ecologically valid and produce transferable results.

\section{Methods}

\subsection{Participants}

Eight skilled football players (age $20.7 \pm 1.2$ years, height $1.74 \pm 0.03 \mathrm{~m}$, mass $71.8 \pm 7.9 \mathrm{~kg}$ ) were recruited from the University 1 st football and futsal teams. All futsal players had a history as a football player prior to University and all players recruited had $9 \pm 4$ years experience of club level football. Players were competing in the British Universities and Colleges Sport Premier North football and futsal leagues and training 3-4 times weekly. None of the subjects had suffered from match-preventive lower limb injuries in the six months prior to testing. All subjects were UK size 8 and right foot dominant, which was determined by asking subjects which side they preferred for kicking. During the test, subjects wore the same brand of new football socks to prevent the socks from altering the subjects' sensation of the boot and ball.

\subsection{Ethics}

The investigation received ethical clearance from the institutional ethics committee and each participant provided written informed consent in accordance with the requirements of the Helsinki Declaration for research using human participants.

\subsection{Football boots}

Two UK size 8 Umbro football boot prototype models were developed for the test (Fig. 1). Fit was ensured from verbal feedback and palpation prior to testing. Both prototypes had the same firm ground outsole similar to the Umbro UX Accuro Pro. The uppers were also the same in terms of central lacing and the smooth white synthetic material. The boots only differed in upper padding; one boot had no padding $(0 \mathrm{~mm})$ and the other had $6 \mathrm{~mm}$ of Poron foam padding (6 mm, XRD 12236 [10]; Fig. 1).

\subsection{Experimental design}

Subjects participated in two sessions each of $2 \mathrm{~h}$ duration and separated by 5-7 days. Both the padded and unpadded upper boots were tested in each session with the boot order randomised across subjects and sessions. Inter-session reliability assessment was based on the 0 -mm padding condition results from the two sessions, while the effect of padding assessment was based on the 0 -mm padding results from

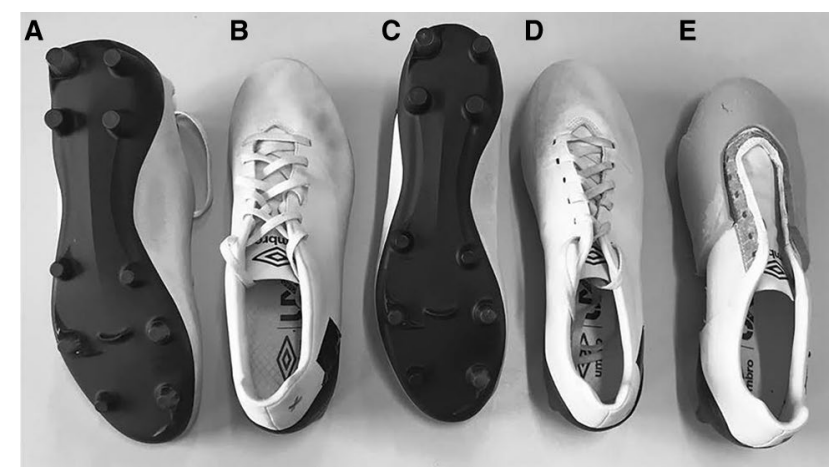

Fig. 1 Plantar and dorsal views of the: a, b 0 -mm padded boot; and c-e $6 \mathrm{~mm}$ Poron foam padded boot where $\mathbf{e}$ is a dorsal view with the upper reversed to illustrate the extent of the padding 
one session and 6-mm padding results from the other session (randomised across subjects). Each session comprised a standardised warm-up and familiarisation of each drill performed in the subject's own football boots prior to testing. The testing involved three drills—dribbling, short passing and long passing, which were completed in this order throughout. Two familiarisation runs and six recorded trials of the dribbling drill were completed. Five familiarisation passes and eight recorded trials were performed for both short and long passes. All drills were completed with the first test boot before the subject changed to the second test boot and the drills repeated. Two subjects were tested in each session and alternated trials throughout to minimise fatigue.

\subsection{Test setup}

The same ball, an Adidas Brazuca football (Adidas, Herzogenaurach, Germany; $22 \mathrm{~cm}$ diameter, $0.43 \mathrm{~kg}$ mass, $0.9 \mathrm{bar}$ pressure), was used in all sessions. Pressure was tested before and after each session with no measurable change during the session. Tests were performed on the same outdoor third generation artificial pitch (LigaTurf RS + CoolPlus 260, Polytan, Burgheim, Germany). In brief, the pitch had a $25 \mathrm{~mm}$ in situ rubber shock pad, the carpet fibres were $60 \mathrm{~mm}$ monofilament polyethylene and the infill comprised $15 \mathrm{~kg} \mathrm{~m}^{-2}$ sand and $15 \mathrm{~kg} \mathrm{~m}^{-2}$ rubber crumb giving a total infill height of $41 \mathrm{~mm}$. Pitch testing using the FIFA Quality Concept methodologies [11], gave a force reduction of $69.6 \pm 1.5 \%$, vertical deformation of $11.4 \pm 0.5 \mathrm{~mm}$ and rotational resistance of $31.9 \pm 1.3 \mathrm{Nm}$. Tests were only performed under dry conditions.

The dribbling test setup incorporated two tasks: loop turn dribbling and zig-zag dribbling (Fig. 2). Subjects started by performing eight loop dribbles. After the loop turns the path carried on into eight zig-zag cuts. Cones were placed in two parallel lines $1.6 \mathrm{~m}$ apart. Cones within each line were placed $3 \mathrm{~m}$ apart. The dimensions were chosen based on pilot testing; with sufficient turns to gather repeated data sets for analysis without inducing fatigue to subjects and appropriately narrow turns to challenge the subject's dribbling ability. Subjects were instructed to complete the drill as fast as possible without losing ball control. They were free to use any part of either foot to control the ball.

Subjects would only complete a dribbling trial when their heart rate (SUUNTO X6HR and Memory Belt chest straps; SUUNTO, Vantaa, Finland) fell below 110 beats $\min ^{-1}$
Fig. 2 Planar view of the test setup for: a dribbling drill, b short passing drill, and $\mathbf{c}$ long passing drill
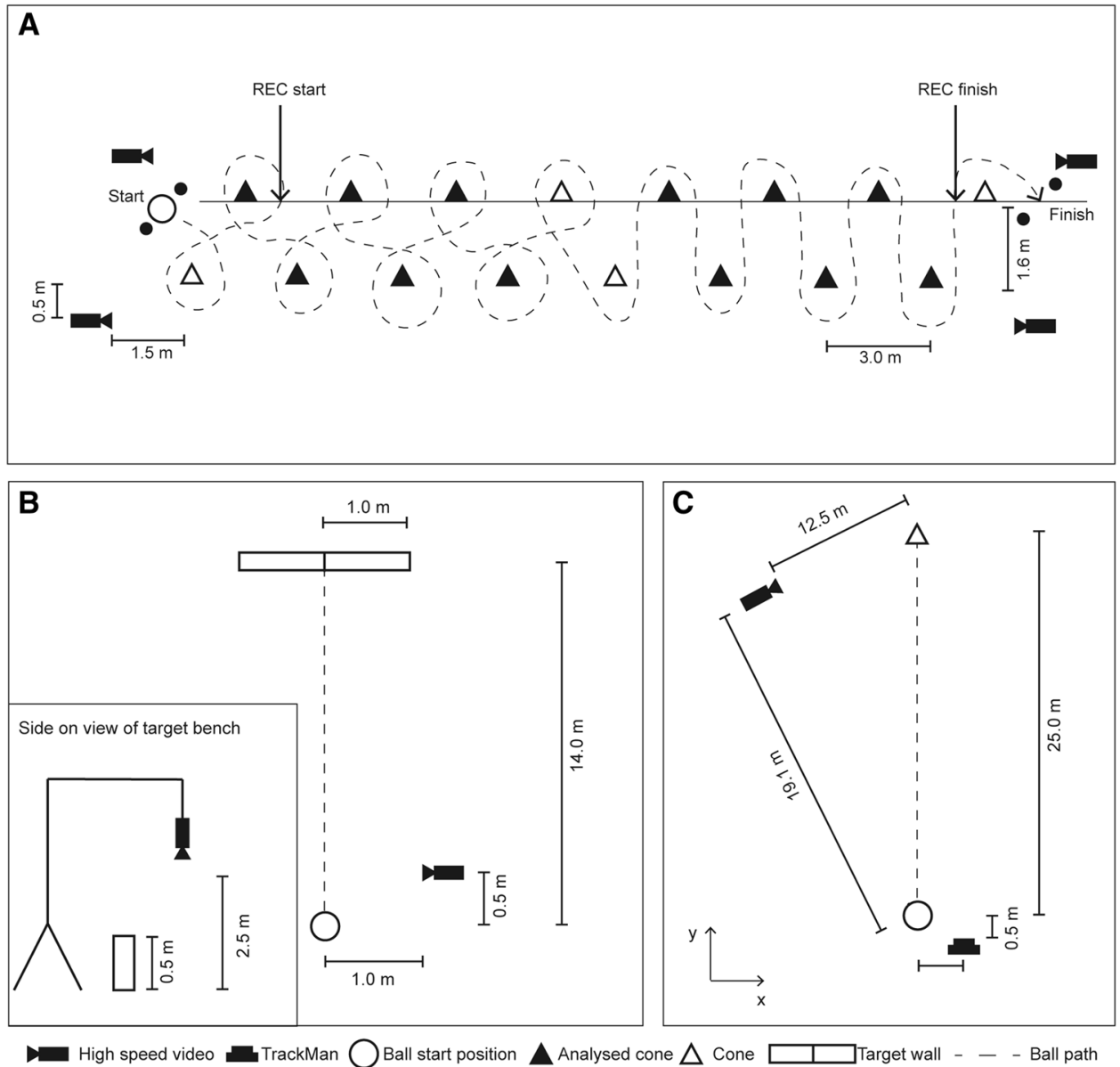
(within their recovery zone according to Fox \& Haskell) [12] and they reported themselves ready. If subjects rated a dribbling trial poor or very poor on a 5-point Likert scale then the trial was repeated. The number of trials required to achieve the six successful dribbling trials was recorded.

For short passing, subjects passed a stationary ball towards the centre of a $2 \mathrm{~m}$ wide and $0.5 \mathrm{~m}$ high wall located $14 \mathrm{~m}$ from the initial ball position (Fig. 2). Subjects were instructed to "pass the ball with the inside of the foot along the ground with no bounce imagining passing to a teammate in the centre mid of the pitch to maintain position. The ball therefore needs to be passed at a match realistic speed'. Short passing distance was validated based on Opta Sportsdata Ltd. (London, UK) data from a single FA Premier League match [13] (total count =286; Manchester City Football Club, 2011). The Premier League data gave a mean passing length of $13.8 \pm 6.2 \mathrm{~m}$ and based on this a length of $14 \mathrm{~m}$ was selected.

For long passing, subjects performed an airborne pass from a stationary ball starting position to a cone placed $25 \mathrm{~m}$ away (Fig. 2). Subjects were instructed to "pass an airborne ball ( $\geq 1 \mathrm{~m}$ above the ground during flight) with the instep of the foot to reach the marked spot when first bouncing on the ground'. The imitated game scenario explained to the subject was 'the midfielders deep pass to the winger/striker running in behind the opponent defence'. Subjects used a repeated but self-selected run up. The five practice passes were used to determine their preferred run up pattern. Long passing distance was validated based on Opta Sportsdata Ltd. (London, UK) data from a single FA Premier League match [13] (total count $=43$ ). The mean pass length of airborne passes was $28.3 \pm 12.0 \mathrm{~m}$ which included passes of $>40 \mathrm{~m}$, which are likely to represent goal kicks, crosses and clearances. Thus a length of $25 \mathrm{~m}$ was selected.

After each pass trial (short and long passing), subjects were asked to rate their technique and the ball speed on 5 -point Likert scales ranging from very poor to very good and much lower than match speed to much faster than match speed. If technique was rated poor or very poor or ball velocity was not rated as match speed then the trial was retaken. The total number of trials required to achieve eight successful passing trials was recorded.

\subsection{Analysis of measures}

To assess dribbling performance number of touches, total time to complete drill and maximum lateral deviation from the cones were analysed. Number of touches and time to complete drill were determined using a chest mounted GoPro HERO4 Black camera $(120 \mathrm{~Hz}, 1280 \times 720)$. The number of touches was manually determined in GoPro Studio (Version 2.5.7, GoPro Inc., San Mateo, CA). By placing the left row of cones on the side line (white line on the ground), it allowed start and finish point to be assessed between first passing the white line ( 2 nd cone) and first passing the white line at the final cone (16th cone). Using alternative start and finish points (Fig. 2a) was chosen to avoid acceleration into the drill and deceleration out of the drill to impact the scores. Subjects were, however, told that the entire drill was examined.

Maximum lateral ball deviation from the cone when turning was assessed (cones marked in black; Fig. 2a) using four static GoPro HERO4 Black cameras $(240 \mathrm{~Hz}, 1280 \times 720$ pixels, barrel distortion $=2.1 \%$ ) positioned perpendicular to and $0.5 \mathrm{~m}$ wider than the cone line and $1.5 \mathrm{~m}$ behind the first cone (Fig. 2a). Mean pixel size for the furthest cones assessed demonstrated a resolution of $1.0 \pm 0.1 \mathrm{~mm}^{\text {pixels }}{ }^{-1}$. Videos were analysed in Image-Pro Analyzer (Version 7.0, Media Cybernetics, Inc., Rockville, MD). Direct linear transformation (DLT) was applied to the lateral deviation point measures to convert points from the image plane reference to the object space reference frame to obtain the offset distance. DLT accuracy levels were $0.012 \pm 0.009 \mathrm{~m}$ along the $\mathrm{x}$-axis (perpendicular to the row of cones) and $0.051 \pm 0.038 \mathrm{~m}$ along the $y$-axis (following the row of cones). DLT analysis was performed in MATLAB (The MathWorks, Inc., Natick, MA) based on the method of Woltring and Huiskes [14] for 2-D camera recordings (http://isbweb.org/software/movan al.html). Any turn where the ball hit the cone or deviated outside the calibration zone was excluded from the analysis.

To assess short passing performance ball velocity and offset from target were measured. Ball velocity was assessed using 2D high-speed video of the initial ball movement after foot contact using a CASIO EX-FH1000 camera (Casio Computer Co., Tokyo, Japan) $(420 \mathrm{~Hz}, 230 \times 170$ pixels, barrel distortion $=<0.1 \%$ ). The camera was placed $0.5 \mathrm{~m}$ in front of the initial ball position with $1 \mathrm{~m}$ setback to record ball velocity (Fig. 2b) allowing a resolution of $4 \pm 1 \mathrm{~mm}$ pixels $^{-1}$. Passing accuracy for short passing was assessed using a GoPro HERO4 Black camera $(240 \mathrm{~Hz}, 1280 \times 720$ pixels) placed on a tripod allowing aerial view of the ball impact on the bench (Fig. 2b). The camera was placed $3 \mathrm{~m}$ above the centred target line on the bench allowing a resolution of $1.0 \pm 0.1 \mathrm{~mm}$ pixels ${ }^{-1}$. All video analysis was conducted using Image-Pro Analyzer. Any short pass that landed outside the calibration zone was excluded from the analysis.

To assess long passing performance ball velocity, radial offset, $\mathrm{x}$-axis offset (perpendicular to kicking direction, Fig. 2) and y-axis offset (kicking direction, Fig. 2) from the target were measured. Ball velocity was assessed using a TrackMan Football system (TrackMan Golf, Vedbaek, Denmark). The TrackMan system was positioned $3 \mathrm{~m}$ behind and $0.5 \mathrm{~m}$ to the right as all subjects were right foot dominant. Accuracy was assessed with a GoPro HERO4 Black camera $(240 \mathrm{~Hz}, 1280 \times 720$ pixels $)$ placed 
on a tripod $1.6 \mathrm{~m}$ above the ground at a $15^{\circ}$ tilt with the target cone in the centre point of the camera. Videos were analysed in Image-Pro Analyzer. DLT was then applied to the offset point measures to convert points from the image plane reference to the object space reference frame to obtain the real world offset distance. DLT accuracy was $0.045 \pm 0.036 \mathrm{~m}$ along the $x$-axis and $0.041 \pm 0.036 \mathrm{~m}$ along the $y$-axis. Any long pass that landed outside the calibration zone was excluded from the analysis.

\subsection{Statistical analysis}

Statistical analysis was carried out using SPSS software (Version 23.0; SPSS Inc., Chicago, IL) with significance set at $P \leq 0.05$ throughout. The mean values for each subject were assessed using non-parametric Wilcoxon's matched pair tests to analyse the effect of upper padding on the 12 dribbling, short passing and long passing performance variables. Non-parametric tests were applied due to violation of sphericity.

To assess the test-retest reliability of the protocol, relative and absolute reliability of the 12 performance variables were examined. The magnitude of relative reliability was determined by the two-way random effect intraclass correlation coefficient $\left(\mathrm{ICC}_{2,1}\right.$; absolute agreement definition) using the mean subject scores for each of the two 0 -mm padding sessions (Weir [15]). Values were interpreted based on the clinical significance levels suggested by Cicchetti [16]. Data was log-transformed due to heteroscedasticity as suggested by Vaz et al. [17] and Weir [15]. Absolute reliability was assessed using standard error of measurement (SEM) and the smallest real difference (SRD) derived from the intraclass correlation coefficients following the methods explained by Weir [15].

\section{Results}

\subsection{Effect of upper padding on dribbling performance}

No dribbling trials had to be repeated due to low subject rating, while the number of excluded turns was consistently low $(\sim 2 \%)$ across all conditions (Table 1$)$. The total time that it took players to complete the dribbling drill showed no significant difference between padding conditions $(0 \mathrm{~mm}$ : $29.2 \pm 1.5 \mathrm{~s} ; 6 \mathrm{~mm}: 29.1 \pm 1.7 \mathrm{~s}, P=0.649)$ despite excellent relative reliability $\left(\mathrm{ICC}_{2,1}=0.879\right)$ and a low absolute reliability $(\mathrm{SRD}=1.4 \mathrm{~s})$. Total number of touches also showed no significant difference between padding conditions $(0 \mathrm{~mm}$ : $54.4 \pm 7.0 ; 6 \mathrm{~mm}: 54.1 \pm 5.8, P=0.652 ; \mathrm{ICC}_{2,1}=0.965$, $\mathrm{SRD}=3.4 \mathrm{~mm}$ ). Similarly, radial offset demonstrated no significant difference between padding conditions for either turn type (Table 2).

\subsection{Effect of upper padding on short passing performance}

To obtain a total of 64 short passes, two or less passes were repeated due to subject rating for each condition, while no long passes had to be excluded due to the pass not landing in the calibration zone (Table 3 ). There was no significant difference in ball velocity for the short passing between padding conditions (0 mm: $20.6 \pm 1.3 \mathrm{~m} \mathrm{~s}^{-1} ; 6 \mathrm{~mm}: 20.5 \pm 1.3 \mathrm{~m} \mathrm{~s}^{-1}$, $\left.P=0.139 ; \mathrm{ICC}_{2,1}=0.539, \mathrm{SRD}=1.3 \mathrm{~m} \mathrm{~s}^{-1}\right)$. A total of 64 passes were performed in each boot. 29 passes ended right of target in the $0-\mathrm{mm}$ boot and 30 passes in the $6 \mathrm{~mm}$ and therefore 35 and 34 ended left of target in the $0-\mathrm{mm}$ and 6-mm boot, respectively. The mean offset was not significantly different between padding conditions $(0 \mathrm{~mm}$ : $-0.20 \pm 0.18 \mathrm{~m} ; 6 \mathrm{~mm}:-0.36 \pm 0.22 \mathrm{~m}, P=0.627 ; \mathrm{ICC}_{2,1}$ $=0.627, \mathrm{SRD}=0.22 \mathrm{~m})$.

Table 1 Summary of the number of dribbling trials and turns completed and assessed

\begin{tabular}{|c|c|c|c|c|c|c|c|c|}
\hline \multirow[t]{2}{*}{ Assessment } & \multirow[t]{2}{*}{ Boot } & \multicolumn{3}{|c|}{ Dribbling trials } & \multicolumn{3}{|c|}{ Dribbling turns } & \multirow[t]{2}{*}{ Total assessed } \\
\hline & & $\begin{array}{l}\text { Total } \\
\text { com- } \\
\text { pleted }\end{array}$ & $\begin{array}{l}\text { Total repeated } \\
\text { due to subject } \\
\text { rating }\end{array}$ & Total assessed & $\begin{array}{l}\text { Total } \\
\text { com- } \\
\text { pleted }\end{array}$ & $\begin{array}{l}\text { Total excluded due to } \\
\text { ball bouncing off cone }\end{array}$ & $\begin{array}{l}\text { Total excluded due to } \\
\text { ball leaving calibration } \\
\text { zone }\end{array}$ & \\
\hline \multirow[t]{2}{*}{$0-0 \mathrm{~mm}$} & S1 & 48 & 0 & 48 & 576 & 9 & 1 & 566 \\
\hline & S2 & 48 & 0 & 48 & 576 & 12 & 0 & 564 \\
\hline \multirow[t]{2}{*}{$0-6 \mathrm{~mm}$} & $0 \mathrm{~mm}$ & 48 & 0 & 48 & 576 & 10 & 0 & 566 \\
\hline & $6 \mathrm{~mm}$ & 48 & 0 & 48 & 576 & 12 & 0 & 564 \\
\hline
\end{tabular}

S1 session 1, S2 session 2 
Table 2 Performance variable results for the 0 - and $6-\mathrm{mm}$ padded boots and relative and absolute test-retest reliability scores for repeated 0 - $\mathrm{mm}$ padded boot test sessions

\begin{tabular}{|c|c|c|c|c|c|c|c|}
\hline Skill & Variable & $\begin{array}{l}\text { Padding } \\
\text { thickness } \\
(\mathrm{mm})\end{array}$ & Mean \pm SD & $P$ value & $\mathrm{ICC}_{2,1}$ & SEM & SRD \\
\hline \multirow[t]{12}{*}{ Dribbling } & \multirow[t]{2}{*}{ Time (s) } & 0 & $29.2 \pm 1.5$ & \multirow[t]{2}{*}{.649} & \multirow[t]{2}{*}{.879} & \multirow[t]{2}{*}{ \pm 0.5} & \multirow[t]{2}{*}{ \pm 1.4} \\
\hline & & 6 & $29.1 \pm 1.7$ & & & & \\
\hline & \multirow[t]{2}{*}{ Total touches (n) } & 0 & $54.4 \pm 7.0$ & \multirow[t]{2}{*}{.652} & \multirow[t]{2}{*}{.965} & \multirow[t]{2}{*}{ \pm 1.2} & \multirow[t]{2}{*}{ \pm 3.4} \\
\hline & & 6 & $54.1 \pm 5.8$ & & & & \\
\hline & \multirow[t]{2}{*}{ Turn offset R (m) } & 0 & $0.62 \pm 0.10$ & \multirow[t]{2}{*}{632} & \multirow[t]{2}{*}{.453} & \multirow[t]{2}{*}{ \pm 0.03} & \multirow[t]{2}{*}{ \pm 0.08} \\
\hline & & 6 & $0.60 \pm 0.10$ & & & & \\
\hline & \multirow[t]{2}{*}{ Turn offset L (m) } & 0 & $0.79 \pm 0.10$ & \multirow[t]{2}{*}{694} & \multirow[t]{2}{*}{.679} & \multirow[t]{2}{*}{ \pm 0.04} & \multirow[t]{2}{*}{ \pm 0.12} \\
\hline & & 6 & $0.78 \pm 0.09$ & & & & \\
\hline & \multirow[t]{2}{*}{ Zig-zag offset R (m) } & 0 & $0.81 \pm 0.20$ & \multirow[t]{2}{*}{.373} & \multirow[t]{2}{*}{.220} & \multirow[t]{2}{*}{ \pm 0.08} & \multirow[t]{2}{*}{ \pm 0.23} \\
\hline & & 6 & $0.78 \pm 0.15$ & & & & \\
\hline & \multirow[t]{2}{*}{ Zig-zag offset L (m) } & 0 & $0.71 \pm 0.24$ & \multirow[t]{2}{*}{.580} & \multirow[t]{2}{*}{.518} & \multirow[t]{2}{*}{ \pm 0.12} & \multirow[t]{2}{*}{ \pm 0.33} \\
\hline & & 6 & $0.69 \pm 0.21$ & & & & \\
\hline \multirow[t]{4}{*}{ Short passing } & \multirow[t]{2}{*}{ Velocity $\left(\mathrm{m} \mathrm{s}^{-1}\right)$} & 0 & $20.6 \pm 1.3$ & .539 & .853 & \pm 0.5 & \pm 1.3 \\
\hline & & 6 & $20.5 \pm 1.3$ & & & & \\
\hline & Offset directional (m) & 0 & $-0.20 \pm 0.18$ & .627 & .765 & \pm 0.08 & \pm 0.22 \\
\hline & & 6 & $-0.36 \pm 0.22$ & & & & \\
\hline Long passing & Velocity $\left(\mathrm{m} \mathrm{s}^{-1}\right)$ & 0 & $19.3 \pm 1.1$ & .731 & .957 & \pm 0.2 & \pm 0.6 \\
\hline & & 6 & $19.2 \pm 0.7$ & & & & \\
\hline & Radial offset (m) & 0 & $2.42 \pm 0.46$ & .547 & .303 & \pm 0.31 & \pm 0.86 \\
\hline & & 6 & $2.53 \pm 0.66$ & & & & \\
\hline & $x$-axis offset (m) & 0 & $-0.26 \pm 1.03$ & .260 & .339 & \pm 0.35 & \pm 0.97 \\
\hline & & 6 & $-0.03 \pm 1.00$ & & & & \\
\hline & $y$-axis offset (m) & 0 & $0.35 \pm 1.38$ & .335 & .414 & \pm 0.68 & \pm 1.89 \\
\hline & & 6 & $0.09 \pm 1.31$ & & & & \\
\hline
\end{tabular}

$I C C_{2,1}$ intraclass correlation coefficient: two-way random effect model (absolute agreement definition), $n$ count, $S D$ standard deviation, $S E M$ standard error of measurement $=\mathrm{SD} \times \sqrt{1-\mathrm{ICC}_{2,1}}, S R D$ Smallest real difference at $95 \%$ confidence intervals $=\operatorname{SEM} \times 1.96 \times \sqrt{2}$

Table 3 Summary of the number of short and long passes completed and assessed

\begin{tabular}{|c|c|c|c|c|c|c|}
\hline Drill & Assessment & Boot & $\begin{array}{l}\text { Total com- } \\
\text { pleted }\end{array}$ & $\begin{array}{l}\text { Total repeated due to } \\
\text { subject rating }\end{array}$ & $\begin{array}{l}\text { Total excluded due to ball leav- } \\
\text { ing calibration zone }\end{array}$ & Total assessed \\
\hline \multirow[t]{4}{*}{ Short passing } & \multirow[t]{2}{*}{$0-0 \mathrm{~mm}$} & $\mathrm{~S} 1$ & 65 & 1 & 0 & 64 \\
\hline & & $\mathrm{S} 2$ & 64 & 0 & 0 & 64 \\
\hline & \multirow[t]{2}{*}{$0-6 \mathrm{~mm}$} & $0 \mathrm{~mm}$ & 66 & 2 & 0 & 64 \\
\hline & & $6 \mathrm{~mm}$ & 65 & 1 & 0 & 64 \\
\hline \multirow[t]{4}{*}{ Long passing } & \multirow[t]{2}{*}{$0-0 \mathrm{~mm}$} & $\mathrm{~S} 1$ & 67 & 3 & 0 & 64 \\
\hline & & $\mathrm{S} 2$ & 67 & 3 & 0 & 64 \\
\hline & \multirow[t]{2}{*}{$0-6 \mathrm{~mm}$} & $0 \mathrm{~mm}$ & 66 & 2 & 0 & 64 \\
\hline & & $6 \mathrm{~mm}$ & 68 & 4 & 0 & 64 \\
\hline
\end{tabular}

S1 session 1, S2 session 2

\subsection{Effect of upper padding on long passing performance}

To obtain a total of 64 long passes, four or less passes were repeated due to subject rating for each condition, while no long passes had to be excluded due to the pass not landing in the calibration zone (Table 3). Similarly, there were no significant differences in any of the three offset measures (radial, $x$-axis and $y$-axis) between padding conditions for the long passing (Table 3). A wide spread in the offset was observed for both boots with no obvious visual tendencies or variance (Table 3 ). Again, there was no significant 
difference in ball velocity between padding conditions $(0$ mm: $19.3 \pm 1.1 \mathrm{~m} \mathrm{~s}^{-1} ; 6 \mathrm{~mm}: 19.2 \pm 0.7 \mathrm{~m} \mathrm{~s}^{-1}, P=0.731$; $\mathrm{ICC}_{2,1}=0.731, \mathrm{SRD}=0.6 \mathrm{~m} \mathrm{~s}^{-1}$ ).

\section{Discussion}

This study aimed to assess the effect of football boot upper padding on a player's dribbling and passing performance and at the same time confirm the test-retest reliability of the protocol applied. No significant differences were seen between the two padding conditions $(0$ and $6 \mathrm{~mm})$ in any of the 12 performance measures.

Similarly to the current results, the only previous study to investigate the effect of football boot design on dribbling and passing performance also reported no significant differences in dribbling number of touches or long passing radial offset, although there was a significant difference in dribbling time [6]. However, dribbling time was measured using a light gate system and previous studies have indicated reliability issues when using such a system for the timing of short sprints [18, 19]. Also, the boots used by Sterzing et al. [6] were both commercially available and differed in many design features, e.g. upper material, fit, lacing and mass and, therefore, little can be concluded about the effect of individual design features from their results. Regardless, the results of both this study and Sterzing et al. [6] indicate that, in general, boot design may not be critical for touch control as long as the design replicates current commercial boot design trends. More specifically, this study also indicates that designers can produce boots with additional smooth padding of up to $6 \mathrm{~mm}$ of Poron foam without negatively impacting dribbling and passing performance, providing more freedom in the design of the boot. However, this study only assessed the effect of upper padding on dribbling and passing performance. Whether upper padding impacts other performance metrics, e.g. shooting, remains unknown.

Assessing test-retest reliability is a common method to validate test protocols $[8,16,20]$. Excellent relative reliability scores were confirmed for more than one performance measure for each drill. Absolute reliability scores showed small SRDs around the mean demonstrating good ability to detect differences in performance between football boots. Thus, the protocol developed in this study has the potential to benefit future research investigating the effect of other boot (or ball/surface) design features on dribbling and passing performance.

Defining appropriate levels to identify differences for the dribbling and passing performance outcome is complex and multifactorial. It is important to assess factors in relation to one another as improved performance of one factor may cause worsening of another and, therefore, neutralise the overall performance. For assessing dribbling performance, the current test setup was shown to be able to detect a change in performance of $1.4 \mathrm{~s},(4.7 \%$ of the mean drill completion time) and 3.4 touches ( $6.4 \%$ of the mean count of touches). The measure of lateral deviation from cones was more sensitive to change in performance when assessing loop turns ( $>16 \%$ change) compared to zig-zag turns $(>47 \%$ change). Sterzing et al. [6] obtained significant difference in completion time (boot 1: $7.31 \pm 0.63 \mathrm{~s}$; boot 2: $7.07 \pm 0.69 \mathrm{~s}, 3.3 \%$ change) using light gates, which, despite the variation in drill setup and assessment method, is below the SRD value obtained in this study of $4.7 \%$. Count of touches did, however, not vary between boots ( $<1$ touch; $3.5 \%$ difference) in the study by Sterzing et al. [6], matching sensitivity levels of $6.4 \%$ found in the current study. It should be emphasised that, if significant differences are found, all measures in the multifactorial analysis of change in performance must be taken into consideration to understand how design parameters can impact performance.

The key performance measure for passing is offset from target assuming that the ball velocity is appropriate. This study demonstrated the ability to detect differences between boot designs of $0.22 \mathrm{~m}$ offset for short and $0.86 \mathrm{~m}$ radial offset for long passes. When performing a 14-m flat pass to a team mate, an offset of $0.4 \mathrm{~m}$ from the player should be within the reach of the team mate and a sensitivity of $0.22 \mathrm{~m}$ is therefore able to detect performance impacting changes of short passing accuracy. The receiving player has a longer time to adapt to the ball when receiving a long airborne pass. The direction of the offset does, however, matter. A small offset along the line of the pass (the $y$-axis in this study) can be accommodated by the receiver by adjusting ball control technique, whilst a similar magnitude offset in the lateral direction may be harder for the receiving player to accommodate and lead to an increased risk of a missed pass.

Past literature has used widely varying passing lengths without arguing for the length chosen. This study is the first to critically assess the passing distances in the short and long passing tests applied. Match data from the FA Premier League was used to determine common short and long passing lengths in modern, professional football. Match analysis is growing and more data are available to researchers. This new information should be used when constructing test setups to reproduce football actions with ecological relevance.

\subsection{Limitations}

An important factor, which will highly impact the outcome of human testing, is the technical level of the subjects recruited [21, 22]. Higher technical level leads to reduced intra-subject variability in technique. This study assessed skilled university players and future research should aim to include subjects at an equivalent or higher level to maintain reliability scores within the values obtained in this study. 
No boot 'break in' experience or longer term adaptation period for players was included within the test protocol. It should be acknowledged that both short- and long-term changes in performance may occur when a player is exposed to a new football boot although neither were the focus of this study.

\section{Conclusion}

No significant difference was found between the 0 - and 6-mm padded upper boots for any of the 12 measures used to assess the difference in performance during dribbling, short and long passing. This supports an element of design freedom in the addition of smooth padding, of up to $6 \mathrm{~mm}$ Poron foam, to football boot uppers without having a negative effect on dribbling and passing performance.

The protocol demonstrated acceptable test-retest reliability for a multifactorial assessment of dribbling, short passing and long passing performance in skilled male players. This offers researchers and football boot manufacturers a useful tool to assess football boot designs.

Open Access This article is distributed under the terms of the Creative Commons Attribution 4.0 International License (http://creativeco mmons.org/licenses/by/4.0/), which permits unrestricted use, distribution, and reproduction in any medium, provided you give appropriate credit to the original author(s) and the source, provide a link to the Creative Commons license, and indicate if changes were made.

\section{References}

1. Xerfi 2XDIS04 (2015) Sporting goods companies-World. Market analysis-2013-2015 trends-corporate strategies. Groupe Xerfi. http://www.xerfi.com/presentationetude/The-Global-Sport ing-Goods-Industry:the-market_7XDIS04. Accessed 7 Aug 2016

2. Bloomfield J, Polman R, O'Donoghue P (2007) Physical demands of different positions in FA Premier League soccer. J Sports Sci Med 6:63-70

3. Puma SE (2017) Puma-EvoTouch Pro FG men's football boots. http://us.puma.com/en_US/pd/puma-one-chrome-fg-mens-socce r-cleats/pna103986.html?dwvar_pna103986_color=01. Accessed 20 Oct 2017

4. Nike Inc (2017) Nike magista onda II dynamic Fit FG. https:// store.nike.com/gb/en_gb/pd/magista-obra-ii-football-boot/pid11833147/pgid-12192317. Accessed 20 Oct 2017

5. adidas (2017) adidas-Ace $17+$ purecontrol firm ground boots. http://www.adidas.co.uk/ace-17_-purecontrol-firm-ground-boots /S77165.html. Accessed 20 Oct 2017
6. Sterzing T, Müller C, Wächtler T, Milani TL (2011) Shoe influence on actual and perceived ball handling performance in soccer. Footwear Sci 3:97-105. https://doi.org/10.1080/19424 280.2011.552439

7. Ali A, Williams C, Hulse M et al (2007) Reliability and validity of two tests of soccer skill. J Sports Sci 25:1461-1470. https://doi. org/10.1080/02640410601150470

8. Currell K, Jeukendrup PAE (2008) Validity, reliability and sensitivity of measures of sporting performance. Sports Med 38:297316. https://doi.org/10.2165/00007256-200838040-00003

9. Russell M, Kingsley M (2011) Influence of exercise on skill proficiency in soccer. Sports Med Auckl NZ 41:523-539. https://doi. org/10.2165/11589130-000000000-00000

10. Algeos (n.d.) $\mathrm{PORON}^{\circledR} \mathrm{XRD}^{\mathrm{TM}}$ extreme impact protection-physical properties. http://www.poroncomfort.com/documents/2826/ XRD-Standard-Physical-Properties.aspx. Accessed 27 Oct 2017

11. Fédération Internationale de Football Association (2015) FIFA quality concept for football turf. http://www.fifa.com/mm/docum ent/ afdeveloping/pitchequip/fqc_football_turf_folder_342.pdf. Accessed 8 May 2016

12. Fox SM, Haskell WL (1968) Physical activity and the prevention of coronary heart disease. Bull N Y Acad Med 44:950-965

13. Manchester City Football Club (2011) MCFC analytics. In: MCFC analytics 2011. http://www.mancity.com/mcfcanalytics. Accessed 8 Feb 2015

14. Woltring HJ, Huiskes HWJ (1990) Stereophotogrammetry. In: Berme N, Cappozzo A (eds) Biomechanics of human movement: applications in rehabilitation, sports and ergonomics. Worthington, Columbus, pp 108-127

15. Weir JP (2005) Quantifying test-retest reliability using the intraclass correlation coefficient and the SEM. J Strength Cond Res Natl Strength Cond Assoc 19:231-240. https://doi. org/10.1519/15184.1

16. Cicchetti DV (1994) Guidelines, criteria, and rules of thumb for evaluating normed and standardized assessment instruments in psychology. Psychol Assess 6:284-290

17. Vaz S, Falkmer T, Passmore AE et al (2013) The case for using the repeatability coefficient when calculating test-retest reliability. PLoS one. https://doi.org/10.1371/journal.pone.0073990

18. Altmann S, Spielmann M, Engel FA et al (2017) Validity of single-beam timing lights at different heights. J Strength Cond Res 31:1994-1999. https://doi.org/10.1519/JSC.0000000000001889

19. Cronin JB, Templeton RL (2008) Timing light height affects sprint times. J Strength Cond Res 22:318-320. https://doi.org/10.1519/ JSC.0b013e 31815fa3d 3

20. Lexell JE, Downham DY (2005) How to assess the reliability of measurements in rehabilitation. Am J Phys Med Rehabil Assoc Acad Physiatr 84:719-723

21. Anderson DI, Sidaway B (1994) Coordination changes associated with practice of a soccer kick. Res Q Exerc Sport 65:93-99. https ://doi.org/10.1080/02701367.1994.10607603

22. Manolopoulos E, Papadopoulos C, Kellis E (2006) Effects of combined strength and kick coordination training on soccer kick biomechanics in amateur players. Scand J Med Sci Sports 16:102110. https://doi.org/10.1111/j.1600-0838.2005.00447.x 